\title{
A predictive model for the adjustment of violin bows
}

\author{
Frédéric Ablitzer ${ }^{(1)}$ \\ frederic.ablitzer.etu@univ-lemans.fr \\ Nicolas Dauchez ${ }^{(2)}$ \\ nicolas.dauchez@supmeca.fr \\ Jean-Pierre Dalmont ${ }^{(1)}$ \\ jean-pierre.dalmont@univ-lemans.fr \\ avenue Olivier Messiaen, 72085 LE MANS Cedex 9, France
}

(1) Laboratoire d'Acoustique de l'Université du Maine (UMR CNRS 6613)

${ }^{(2)}$ Laboratoire d'Ingénierie des Systèmes Mécaniques et des Matériaux Supméca, 3 rue Fernand Hainaut, 93407 ST-OUEN Cedex, France

PACS numbers: 43.75.De

(dated: August 26, 2011) 


\begin{abstract}
A finite element model aimed at predicting the in-plane and out-of-plane static mechanical behavior of a tightened bow is developed. It takes into account the prestress due to hair tension and the nonlinear behavior due to large displacements. An non-destructive procedure to determine the input parameters of the model from measurements on a bow is described. Numerical and experimental results are then compared in the case of two bows, showing good agreement between the simulated and measured mechanical behavior. Finally, hair tension and camber are shown to influence the proportion between lateral and vertical compliances of the tightened bow. The model might be used by bow makers for the adjustments of bows during the making process.
\end{abstract}




\section{Introduction}

As an interface between the player's arm and the string, the bow is an all-important element in violin playing. In spite of its apparent simplicity, it is a very elaborate mechanical device, which requires high expertise from bow makers to meet the demand of professional players.

The primary function of a bow (Fig. 1) is to maintain a hair ribbon under tension. This gives little latitude in the choice of the wood, since the stick must remain slender in spite of the high tension it has to withstand [1]. Moreover, the modern bow has a very standard geometry, which could lead to believe that the quality of a bow only hangs on the wood. However, by doing subtle adjustments on the geometry of the stick, bow makers have the ability to draw the best from different wood blanks with various mechanical properties, or even to adapt the playing qualities of a bow to the needs of a specific player. Actually, bow makers work with three main parameters when making a stick:

- wood (density, elasticity, damping);

- taper, which denotes the gradually decreasing thickness along the stick;

- camber, that is the shape of the stick without hair tension.

For the choice of the wood, some bow makers combine their know-how with a scientific approach, by using specific equipments (such as a Lucchimeter [2,3], or Lutherie Tools [4]) to measure some of the wood properties. Regarding taper and camber, however, a specific device aimed at assisting bow makers does not exist at present. The mastery of these conception parameters thus requires a high expertise from the bow maker. The aim of our study is to develop a tool based on a physical model, capable of predicting the mechanical behavior of a bow with regard to its material and geometric characteristics. This study comes within the scope of a research project aimed at supplying instrument makers with dedicated measurement and simulations tools which are affordable and easy-to-use [5].

In most previous studies, the static behavior of the bow has been treated as an in-plane problem $[6,7,8,10]$. However, examining the out-of-plane behavior seems to be relevant as well. Indeed, the bow is often slightly tilted towards the fingerboard in playing [11], which makes the stick bend in both vertical and lateral directions. Moreover, even when the bow is played with the hair flat on the string, the stick may bend laterally in response to small hand movements. Thus, the player probably feels both vertical and lateral compliances when he controls the bow force. Lastly, discussions with bow markers suggest that the proportion between vertical and lateral bending of the stick under tension not only depends on taper, but 
also on the amount of camber. The out-of-plane behavior hence appears to be also considered when making or adjusting a bow. Therefore, it is taken into account in the model developed in this paper.

In section 2 , the three-dimensional finite element model is presented. In section 3 , the experimental procedure to determine the input parameters of the model from measurements on a bow is described and validated. Finally, some tendencies on the behavior of the bow when hair tension and camber vary are given in section 4 .

\section{Model}

To take into account geometric nonlinearity, a large displacement formulation based on the co-rotational approach is adopted. The main steps of the formulation (after Crisfield [12]) are presented below.

\subsection{Co-rotational formulation}

The main idea of the co-rotational formulation is to separate the total displacements of the structure into rigid-body motion and local deformation, as illustrated by Figure 2. The rigidbody motion can be arbitrarily large, while the local deformation is assumed to remain small. To each element is associated a local frame, materialized by base-vectors $\mathbf{e}_{1}, \mathbf{e}_{2}$ and $\mathbf{e}_{3}$ in Figure 2, which translates and rotates with the element.

In the local frame, the displacements caused by deformation are described by a set of local degrees of freedom (DOF) : axial elongation $\left(u_{l}\right)$, torsional angles $\left(\theta_{l 1}\right.$ at node $1 ; \theta_{l 4}$ at node $2)$, bending slopes $\left(\theta_{l 2}, \theta_{l 3}\right.$ at node $1 ; \theta_{l 5}, \theta_{l 6}$ at node 2$)$. These local displacements, gathered in vector $\mathbf{p}_{l}$, are conjugate to local internal efforts $\mathbf{q}_{i l}$ (axial force, torsional moments, and bending moments, respectively). Since the local deformation is small, a linear relationship between local displacements and internal efforts can be written as:

$$
\mathbf{K}_{l} \mathbf{p}_{l}=\mathbf{q}_{i l}
$$

where $\mathbf{K}_{l}$ is the local stiffness matrix $(7 \times 7)$, which remains the same during the analysis, assuming material linearity.

In any deformed configuration of the structure, the local displacements $\mathbf{p}_{l}$ can be computed from the global displacements $\mathbf{p}$, which relate to the global frame and consist of 6 DOF per node (3 translations and 3 rotations). Moreover, it is possible to express a relationship 
between infinitesimal changes of local and global displacements, resp. $\delta \mathbf{p}_{l}$ and $\delta \mathbf{p}$, as:

$$
\delta \mathbf{p}_{l}=\mathbf{F} \delta \mathbf{p}
$$

where $\mathbf{F}$ is the transformation matrix $(7 \times 12)$. Contrary to the local stiffness matrix, $\mathbf{F}$ depends on displacements and has to be computed at each iteration. Knowing the transformation matrix, it is possible to relate the global internal efforts $\mathbf{q}_{i}$ to the local internal efforts $\mathbf{q}_{i l}$ given by Eq. (1). For this, we express the fact that the internal virtual work has to be the same in the global and local frame, which yields:

$$
\mathbf{q}_{i}=\mathbf{F}^{\mathrm{T}} \mathbf{q}_{i l}
$$

The last step in the formulation is to express the tangent stiffness matrix $\mathbf{K}_{t}$. The static equilibrium of the structure is expressed by:

$$
\mathbf{g}=\mathbf{q}_{i}-\mathbf{q}_{e}=\mathbf{0}
$$

where $\mathbf{g}$ is the vector of out-of-balance efforts and $\mathbf{q}_{e}$ the vector of external efforts, which are assumed here to be independent of the displacements. For a given loading $\mathbf{q}_{e}$, the displacements $\mathbf{p}$ that satisfy the static equilibrium are found iteratively. Assuming that the structure at iteration $(i)$ is not in equilibrium and expanding $\mathbf{g}$ in a first-order Taylor series about the current displacements $\mathbf{p}^{(i)}$, Eq. (4) becomes:

$$
\mathbf{g}^{(i)}+\left.\frac{\partial \mathbf{g}}{\partial \mathbf{p}}\right|_{\mathbf{p}^{(i)}} \delta \mathbf{p}^{(i+1)}=\mathbf{0},
$$

where $\delta \mathbf{p}^{(i+1)}$ is the vector of incremental displacements between the current and next iteration, i.e. $\mathbf{p}^{(i+1)}=\mathbf{p}^{(i)}+\delta \mathbf{p}^{(i+1)}$. The tangent stiffness matrix is then:

$$
\mathbf{K}_{t}=\frac{\partial \mathbf{g}}{\partial \mathbf{p}}=\mathbf{F}^{\mathrm{T}} \mathbf{K}_{l} \mathbf{F}+\frac{\partial \mathbf{F}^{\mathrm{T}}}{\partial \mathbf{p}} \mathbf{q}_{i l}
$$

The first term in $\mathbf{K}_{t}$ corresponds to the elasticity of the material, and the second to geometric stiffness, due to the stress field in the structure.

\subsection{Model of bow}

\subsubsection{Assumptions on material}

Wood is a complex material, both anisotropic and inhomogeneous. It is generally described as an orthotropic material, needing 9 independent elastic constants. However, bow makers usually follow the direction of grain when cutting a wood blank and avoid areas of wood that present growth defects $[13,14]$. Consequently, following assumptions can be made: 
- The wood is homogeneous.

- The local reference frame of each beam element coincides with the natural directions of the wood. In particular, the vector $\mathbf{e}_{1}$ is assumed to correspond to the longitudinal direction. Thus, only the longitudinal Young's modulus $E_{L}$ of the wood is needed to account for the flexural and traction/compression behavior, and the shear moduli $G_{R L}$ and $G_{T L}$ for the torsional behavior. A value of $E_{L} / G_{(R T) L}=15$, typical for hardwood, is chosen [15].

As it is a natural material, the horse hair as well is inhomogeneous. Its mechanical properties may vary among the hairs contained in the ribbon, and along the length of an individual hair [16]. As seen by the tightened stick, however, the hair ribbon may be characterized by its longitudinal stiffness, typically of the order $30 \mathrm{~N} / \mathrm{mm}$ [6]. The longitudinal stiffness of the hair ribbon is necessary to account for variations in hair tension as the tightened bow is loaded.

\subsubsection{Assumptions on structure}

Due to the slenderness of the bow stick, Euler-Bernoulli kinematic assumptions are chosen. The influence of shear forces is thus neglected. Only the part of the bow between the front end of the frog and the tip is modeled, as shown by Figure 3. The origin of the global coordinate system is defined as the front end of the frog on the loosened bow. The $x$ axis is oriented over the length of the bow. The $z$ axis is oriented normally to the under surface of the frog. A moving reference frame $\left\{x_{b}, y_{b}, z_{b}\right\}$ is associated to the bow. The $x_{b}$ axis coincides with the direction determined by the endpoints of the hair. In the following, the "vertical" and "lateral" directions correspond to the $z_{b}$ and $y_{b}$ axes, respectively.

The stick is discretized into $N=20$ elements, plus one for the head. The length of each element is chosen such that the decrease in diameter from one node to the next is as identical as possible along the bow. Since the diameter decreases more rapidly when approaching the tip, more elements with smaller size are necessary in this region to meet the criterion (see Fig. 3). As the head is stubby and oriented perpendicular to the grain, the Euler-Bernoulli beam modeling as well as the longitudinal Young's modulus are inappropriate to account for its actual behavior under loading. However, assuming that the deformation of the head is negligible compared to that of the stick, it is represented by a single element with arbitrary but large enough cross-section dimensions (such as a $15 \mathrm{~mm} \times 10 \mathrm{~mm}$ ellipse, for instance).

In this paper, the initial curvature of the stick is assumed to be plane. This assumption 
is satisfied on most bows of respectable quality.

A hair ribbon is usually made up of 150 to 200 hairs. In first approximation, it may be represented by an equivalent single hair. The underlying assumptions are that every hair in the ribbon participate to the force exerted on the stick, and that the resultant force is centered in the width of the ribbon. Actually, as the initial lengths of the hairs may slightly differ, the first assumption is true only when a certain resultant hair tension is reached. For reason of consistency in the co-rotational finite-element formulation, a beam modeling is retained. It takes into account longitudinal stiffness (i.e. possible stretching) and geometric stiffness due to tension. In order to obtain a longitudinal stiffness of $30 \mathrm{~N} / \mathrm{mm}$ for a $65 \mathrm{~cm}$ ribbon with a typical Young's modulus $E_{L}^{h}=5 \mathrm{GPa}$, the dimensions of the rectangular cross-section are fixed to $10 \mathrm{~mm} \times 0.39 \mathrm{~mm}$. As the beam modeling introduces spurious bending stiffness, especially in the lateral direction, the second moments of inertia $I_{y}$ and $I_{z}$ are assigned a sufficiently low value (both $1 \%$ of $I_{y}$ with the actual dimensions). This ensures that only geometric stiffness intervenes in the transverse behavior of the hair. The beam representing the hair is connected to the stick at the tip by a spherical joint, thus disabling the transmission of moments between one body to the other. It is discretized into $N_{h}=10$ elements having the same length.

Alternatively, the ribbon may be modeled by several equivalent hairs, regularly distributed along the width of the end. In this case, each equivalent hair is connected to an intermediary rigid beam at the tip. To facilitate the generation of the mesh, an odd total number of equivalent hairs $n_{h}$ is chosen, keeping one in the middle and adding others on both sides.

\subsection{Steps of the simulation}

A typical use case of the model is to predict the compliance of the tightened bow. To achieve this, the simulation is decomposed into two load steps. Throughout the simulation, the stick is clamped at the frog. In the first step, the end node of the hair corresponding to the attachment to the frog is allowed to translate along the $x$ axis. It is loaded by a force with magnitude $T_{0}$ oriented in the direction of the hair ( $x_{b}$ axis), resulting in backward displacement of the node and straightening of the stick. In the second step, the position of the same node is kept fixed by blocking the translation. The bow, which is in a prestressed state, may be loaded by a force $F_{z}$ at any node of the hair, with an angle $\psi$ relative to the vertical axis of the bow $z_{b}$ (see Fig. 3). This force corresponds to the bow pressure that would be exerted by the player on the string, with the bow eventually tilted. To determine the compliance, an incremental loading is chosen, typically from 0 to $1.6 \mathrm{~N}$ by steps of $0.1 \mathrm{~N}$. 
This allows for numerical differentiation of the force-deflection relationship.

The finite-element model of bow is implemented in Matlab. Each load step is solved iteratively by using a Newton-Raphson procedure. The chosen convergence criterion is $\|\mathbf{g}\|<$ $\varepsilon$ [see Eq. (4)], the value of $\varepsilon$ being chosen very small compared to that of typical external efforts exerted on the structure. In the general case where the hair ribbon is represented by $n_{h}$ equivalent hairs, the total numbers of nodes is $N+1+n_{h} \times\left(N_{h}+2\right)$. As the single equivalent hair approximation $\left(n_{h}=1\right)$ is chosen for most simulations, the mesh typically consists of 33 nodes, each having 6 DOF. When running the simulation on a personal computer, the time to obtain the compliance at all nodes of the hair is approximately 10 minutes, for one value of angle $\psi$. The in-plane finite element model previously developed by the authors [10], thought taking into account geometric nonlinearity as well, is less computationally expensive. The mesh consists of 22 nodes with 3 DOF representing the stick, whereas the behavior of the hair is treated by analytical equations. As a comparison, the simulation with the same input parameters takes about 20 seconds. Thus, the in-plane model will be preferably used when the out-of-plane behavior of the bow has not to be considered, all the more when a large number of simulations is needed (e.g. inverse method, Monte Carlo method).

\section{Experimental procedures}

In this section, we describe the non-destructive procedure to determine the bow parameters. Then, a method to measure the compliance along the bow is presented. The experimental procedures are illustrated here on two students bows (B1 and B2, see section 4, Tab. 3).

\subsection{Determination of bow parameters}

The input parameters of the model are:

- the geometry: camber, vertical and horizontal diameters,

- the properties of the material: Young's modulus of the stick $E_{L}$, Young's modulus of the hair $E_{L}^{h}$,

- the loading: hair tension $T_{0}$, bow force $F_{z}$ at relative abscissa $\gamma$ with tilt angle $\psi$.

Bow parameters include the geometry and the material properties, and in a certain sense the hair tension that characterizes a playing state. These parameters are determined successively, in the order illustrated by Figure 4 . 


\subsubsection{Geometry}

Although the cross-section of the stick could be visually described as round, it is assumed to be oval. The vertical and horizontal diameters are measured at equally spaced abscissas along the stick, by steps of $25 \mathrm{~mm}$. Due to the presence of the wrapping, the portion between 0 and $100 \mathrm{~mm}$ can not be measured. A piecewise cubic interpolation allows for discretization at any abscissas. The diameter of each element is then taken as the mean value of the diameter at its nodes. As seen on Figure 5, the vertical and horizontal diameters only slightly differ, mainly in the first half on the bow.

The camber is determined from a picture of the bow without hair tension, by means of image processing. The bow reposes on two supports, at the frog and at the tip. A backward diffuse lighting is used such as to obtain a well contrasted image. The picture is taken with a Canon OS 300D digital camera equipped with Sigma 18-50 mm F3.5-5.6 DC lens. The focal length is set to $50 \mathrm{~mm}$, at which the lens produces negligible distortion. The resolution, determined by placing a ruler in the same plane as the bow, is comprised between 0.24 and $0.26 \mathrm{~mm} / \mathrm{px}$, depending on the distance between the camera and the bow. The camera is mounted on a tripod, in order to hold the distance constant between successive pictures of a same set of measurements. The end points of the hair at the front end of the frog and at the tip are pointed out manually on the picture. Then, the upper and lower outlines of the stick are detected to obtain its neutral axis. It is approximated by a polynom (see Fig. 6), with an order such that the residual error is below $0.1 \mathrm{~mm}$, i.e. approximately one half of a pixel.

\subsubsection{Material properties and hair tension}

The Young's modulus of the stick $E_{L}$, the hair tension $T_{0}$ and the equivalent Young's modulus of the hair $E_{L}^{h}$ for a given cross-section area are determined successively by an inverse method in 3 steps. For each parameter, a specific loading is imposed on the bow and the deflected shape of the stick is measured on a picture. Then, a simulation with the same loading is performed. Measured and simulated deflected shape are compared and the unknown input

parameter (either $E_{L}, T_{0}$ or $E_{L}^{h}$ ) is found such as to minimize the difference between the two. An illustration of the optimization routine, which is common to the three steps, is illustrated by Figure 7 in the case of the determination of hair tension.

1. To determine the Young's modulus of the stick $E_{L}$, the bow without hair tension is clamped at the frog and loaded at the tip by a vertical force $F_{z}$ [Fig. 4-(1)].

2. The hair tension $T_{0}$ is determined by tightening the bow [Fig. 4-(2)]. 
3. To determine the Young's modulus of the hair $E_{L}^{h}$, the tightened bow is clamped at the frog loaded at the tip by a vertical force $F_{z}$ [Fig. 4-(3)]. With such a loading, the stiffness of the hair counteracts the tendency of the force to slightly increase the distance between the frog and the tip. The hair tension thus increases in proportion to the stiffness of the ribbon, which affects the deflected shape of the stick.

In our experiment, the clamped boundary condition is realized by two metal fingers grasping the bow (see Fig. 8), assuming that the frog is perfectly rigid and bound to the stick. One finger is in contact with the upper surface of the stick at abscissa $x=0 \mathrm{~mm}$ and the other with the under surface of the frog, near its rear end. This solution is preferable to those based on preloading (e.g. using a bar clamp), since an excessive pressure between the frog and the stick might damage the edges of the stick around the mortise. Moreover, since the measured and simulated deflected shape of the stick are compared in reference frame of the bow (see Fig. 7), a slight rigid-body rotation of the bow has no consequence on the results of the procedure. Consequently, compliant pads could be placed between the metal fingers and the bow such as to avoid marking it. The force $F_{z}$ was simply measured by a weighing scale (accuracy $0.01 \mathrm{~g}$ ), which was brought in contact with the tip through an intermediary triangular-shaped part. As the base equipment necessary to apply the procedure (personal computer, digital camera with tripod, weighing scale) is generally already owned by bow makers, the method is easily transferable in workshops, as a tool to assist bow making.

\subsubsection{Uncertainties}

In order to validate the procedure to determine the bow parameters, uncertainties are calculated using the Monte-Carlo method [17]. For all input variables involved in the procedure, a type $\mathrm{B}$ uncertainty is considered. The input variables $x_{i}$ and their uncertainties $\Delta x_{i}$ are listed in Table 1. They have been set according to following considerations:

- Uncertainties on vertical and lateral diameter are assigned to each element. The discretization of the stick is such that the difference in diameter between two consecutive nodes is around $0.2 \mathrm{~mm}$. As the diameter of each element is defined as half between those at its nodes, the resulting discretization error (about $0.1 \mathrm{~mm}$ ) is higher than the accuracy of the digital caliper $(0.02 \mathrm{~mm})$. Thus, it is chosen to represent the uncertainties on diameters. It should be noted that these uncertainties may also represent small variations in Young's modulus along the stick (i.e. inhomogeneity of the wood), as they 
affect bending stiffness $E_{L} I$. For instance, a typical error of $1 \%$ on the diameter of an element is equivalent to a $4 \%$ local variation in Young's modulus.

- The uncertainties on the abscissas and heights of both frog and tip arise from the difficulty to determine accurately the end points of the hair on the picture, because of the width of the ribbon. As the residual error in the characterization of camber by a polynom $(<0.1 \mathrm{~mm})$ is smaller than the uncertainty on frog and tip heights $(1 \mathrm{~mm})$, no local uncertainty is assigned to camber.

- The uncertainty on the force exerted at the tip in steps 1 and 3 is chosen such as to include error due to stress relaxation, which occurs while the deflection of the bow is held constant.

The Monte-Carlo method is applied as follows:

1. A set of random values of input parameters within the interval $\left[x_{i}-\Delta x_{i} \quad x_{i}+\Delta x_{i}\right]$ are generated, considering a uniform distribution.

2. Output parameters $y_{j}\left(E_{L}, T_{0}\right.$ and $\left.E_{L}^{h}\right)$ are determined successively by inverse method, from the same set of input parameters.

3. Steps 1 and 2 are repeated a large number $M$ of times $(M=2000)$.

4. For each output parameter $y_{j}$, the mean value $\bar{y}_{j}$ and standard deviation $\sigma_{j}$ over the $M$ obtained values are calculated.

5. The estimate $Y$ of the output parameter is expressed as $Y_{j}=\bar{y}_{j} \pm k \sigma_{j}$, where $k$ is a coverage factor. Assuming a normal distribution for the output parameters, it is taken here as $k=1.96$, which corresponds to a $95 \%$ confidence interval.

The parameters determined by the procedure are presented in Table 2 with their uncertainties. The value of the Young's modulus of the stick $E_{L}$ is consistent with those typically reported in the literature [18]. As the corresponding uncertainty is rather low (2\%), confrontation with other measurement methods would be helpful to discuss the validity of the experimental procedure. The uncertainty on the hair tension $T_{0}(6 \%)$ is considered satisfactory for using the model as an indirect measurement method. The uncertainty calculated for the Young's modulus of the hair $E_{L}^{h}$ may seem deceptive (22\%). However, it can be explained by the fact that the compliance of the tightened bow is fare more sensitive to the elasticity of the stick than that of the hair. 


\subsection{Measurement of compliance}

The experimental setup used to measure the compliance of the bow is shown on Figure 8. A force transducer (HBM U1A, $10 \mathrm{~N}$ range) is mounted on the mobile part of a height gage and equiped with a touching part. A potentiometric displacement transducer (Meiri PZ12, $100 \mathrm{~mm}$ range), fixed at the steady part of the height gage, is used to measure the vertical translation of the mobile part.

The bow is clamped at the frog and free at its end. The measurement device is placed successively at different abscissas along the bow. At each abscissa, the mobile part is manually translated upward and downward, making the bow deflect. Throughout the measurement, the force applied to the hair is monitored with an oscilloscope such as to avoid excessive loading. The signals delivered by the force and displacement transducers are acquired simultaneously at sampling rate of $200 \mathrm{~Hz}$. For the validation of the model, the in-plane as well as the outof-plane behavior have to be investigated. Thus, a measurement is also carried out with the bow clamped such that the compliance is measured in the lateral direction. In the following, the term "lateral compliance" refers to this configuration, whereas "vertical compliance" corresponds to the configuration visible on Figure 8.

Figure 9 shows typical force-deflection curves obtained with the measurement device. The data points extracted from the acquisition correspond to the loading of the bow only, as a slight hysteresis could be observed between loading and unloading. Regarding the inplane behavior, the evolution of deflection with force is slightly nonlinear. Near the tip, the slope of the force-deflection curve increases with force, whereas if decreases with force in the middle. Moreover, the slope of the lateral force-deflection curve at the tip is higher than in the vertical case. In the following, we define compliance $c$ as the slope of force-deflection

curve, i.e. $c=\frac{\partial u_{z}}{\partial F_{z}}$. For this purpose, the data points are fitted with second order polynoms, which are then differentiated to calculate the compliance.

\section{Discussion}

In this section, the model and the experimental procedures previously described are used to compare the mechanical behavior of two bows. 


\subsection{Description of the bows}

\subsubsection{Bow characteristics}

The experiments are carried out on two student bows of respectable quality, made of Pernambuco. The two bows were selected by a bow-maker, for the similar characteristics of their sticks in terms of overall design, mass and stiffness. They were re-haired and the wire wrapping was made such as to reach mass and center of inertia as close as possible on both bows. Then, one bow was given more camber (bow B2) than the other (bow B1), as visible in Figure 10, with an effort to keep the distribution of camber along the stick the same as possible. Some characteristics of the bows that are straightforward to measure are listed in Table 3. It should be noted that the difference in minimum hair-stick distance between the two bows is $2.4 \mathrm{~mm}$, which indicates a moderate adjustment of camber.

\subsubsection{Studied settings}

Three settings of hair-stick distance at which the bow could be played are chosen. The hairstick distance is set by inserting a small cylinder with adequate diameter between the hair and the stick, in the middle of the bow $(x=325 \mathrm{~mm})$, and gently tightening the bow until the cylinder falls. The minimum hair-stick distance can then be measured with a good accuracy from a picture of the tightened bow. Figure 10 shows the deformed shapes of both bows for the three settings of hair-stick distance, denoted by A1, A2, A3. Whereas the shapes without hair tension significantly differ because of camber adjustment, the shapes of both tightened bows at each setting are almost the same. As the aspect ratio of the figure emphasizes the small differences, they would be hardly perceptible with the naked eye.

The procedure to determine bow parameters described in section 3 is then applied to both bows, to allow simulation with the finite-element model. Having determined the Young's modulus of both sticks, the hair tension corresponding to the three settings is then determined for each bow. The results are shown in Figure 11. As expected, the hair tension increases with the hair-stick distance. Considering that settings A1 and A3 roughly delimit the playing range of each bow, the corresponding range in hair tension varies from 48 to $63 \mathrm{~N}$ on bow $\mathrm{B} 1$, from 65 to $76 \mathrm{~N}$ on bow B2. It clearly appears that a higher camber allows to reach a higher hair tension for the same setting of hair-stick distance, all other geometrical and material properties of the bow being considered identical. Furthermore, these results seem to suggest that the offered range in hair tension is wider when the bow is less cambered ( $15 \mathrm{~N}$ for bow B1 v.s. $11 \mathrm{~N}$ for $\mathrm{B} 2)$. 


\subsection{Comparison between measured and simulated compliance}

\subsubsection{Distribution of compliance along the bow}

For each setting of hair-stick distance, the vertical and lateral compliances are measured on the tightened bows, as described in section 3. The force-deflection relationships are measured at regularly spaced abscissas, by steps of $50 \mathrm{~mm}$ starting from the tip. For each obtained force-deflection curve, the compliance at a typical bow force of $1 \mathrm{~N}$ is then calculated and plotted against corresponding relative abscissa. Figure 12 shows the measured vertical and lateral compliance along bows B1 and B2, for setting A1 (7.8 $\pm 0.1 \mathrm{~mm}$ hair-stick distance). Results of simulations with the bow parameters previously determined are plotted too.

A good agreement between experimental and numerical results is observed. The model manages to reproduce the higher compliance in the lateral direction. This effect is a direct consequence of prestress: when out-of-plane bending of the stick occurs, the hair tension creates an additional bending moment along the stick. This moment acts together with that caused by the force, which makes the deflection higher. This phenomenon is at the bottom of the higher lateral compliance observed on bow B2, for which the hair tension at A1 is higher than on bow B1. For information, the simulated vertical and lateral compliances at the tip of the stick without the hair are $13.0 \mathrm{~mm} / \mathrm{N}$ and $13.2 \mathrm{~mm} / \mathrm{N}$, respectively, on bow B1. On bow B2, they are $13.4 \mathrm{~mm} / \mathrm{N}(+3 \%)$ and $13.7 \mathrm{~mm} / \mathrm{N}(+4 \%)$.

The agreement on the lateral compliance along the bow, except at the tip, should be considered with cautiousness. When applying a force laterally on one side of the ribbon, the number of hairs undergoing deflection actually increases with the force. Hence, the relationship between the force and the deflection is nonlinear, with a decreasing compliance. As the deformation of the ribbon is not taken into account in the model, the agreement declines when considering other forces, e.g. $0.5 \mathrm{~N}$ or $1.5 \mathrm{~N}$. However, the numerical results are probably representative of the lateral compliance felt by the musician when playing with the hair flat on the string.

\subsubsection{Effect of hair tension and camber}

In order to better apprehend how hair tension and camber affect the mechanical behavior of the stick, we now focus on the vertical and lateral compliances measured at the tip $(\gamma=1)$. Figure 13 shows their evolution with hair tension on both bows.

A good agreement between experimental and numerical results is observed on vertical compliance. Regarding lateral compliance, discrepancies of about $10 \%$ are found with the 
single equivalent hair approximation. Yet, a better agreement is observed when using several equivalent hairs to model the ribbon. In this case, the axial stress in the equivalent hairs increases on the side where the force is applied and decreases on the other side. This phenomenon can be observed experimentally simply by "plucking" each side of the ribbon as the tightened bow is loaded and listening to the resulting tone. A higher or lower pitch compared to the unloaded bow indicates a rise or fall in hair tension, respectively. A consequence is that the resultant force exerted on the stick by the ribbon is slightly shifted laterally compared to the case of a single equivalent hair. Therefore, the bending moment created by the hair tension, which enhance the compliance of the stick, is lower, and hence the lateral compliance as well. It should be noted that $n_{h}=3$ hairs are sufficient to cause this phenomenon, the effect of which is small.

In every case, the lateral compliance at the tip is higher than the vertical compliance and increases with hair tension. This is due to the fact that hair tension assists the out-of-plane bending of the stick, as mentioned earlier. On the contrary, the vertical compliance at the tip decreases with hair tension. This can be explained by the fact that the distance between the hair and the neutral axis of the stick increases. Now, the rise in hair tension as the tightened bow is loaded creates a bending moment proportional to this distance. Since this bending moment counteracts the effect of that caused by the external loading, the deflection is reduced when the distance is higher.

For the same hair tension, the lateral compliance at the tip is almost the same on both bows. The difference between bows B1 and B2 is of the order 5\%, which is comparable to the difference on the sticks without the hair (4\%). Thus, it seems that the higher lateral compliance of the tightened bow compared to that of the stick depends on hair tension, but not on camber. The vertical compliance, however, is significantly higher on bow B2 for the same hair tension (between 15 and $20 \%$ depending on hair tension, to be compared a difference of $3 \%$ on the sticks without the hair). Indeed, the same hair tension is reached at a lower hair-stick distance when the bow is more cambered. Thus, the bending moment due to the rise in hair tension is smaller, offering less resistance to loading.

Considering the two bows tightened at the same hair-stick distance, however, the compliance at the tip of bow B2 is higher in both directions. Moreover, the ratio between lateral and vertical compliances as well is higher, as indicated in Table 4. Interpreting these results in terms of playing qualities is far from straightforward, though tempting. However, they might explain the fact that a too much cambered bow is likely to "whip" to one side to the other during playing [19]. 


\section{Conclusion}

A numerical model of violin bow and a procedure for determining its input parameters from measurements have been developed. The essential input parameters are the geometry of the bow, the Young's modulus of the stick, the tension and stiffness of the hair. Their successive determination is possible with rather affordable and easy-to-use equipment. The model is able to predict the mechanical behavior of a tightened bow. It has been shown that the hair tension enhances the bending of the stick under a lateral force. Moreover, increasing the hair tension lowers the deflection of the stick under a vertical force. As a consequence, the gap between vertical and lateral compliances of the bow increases with hair tension. It has also been shown that adding camber allows the player to reach a higher playing hair tension for the same hair-stick distance. Together, both vertical and lateral compliances of the stick are higher. The results are in line with the experience of bow makers, who affirm that camber influences the flexibility of the stick under hair tension.

Thus, the model presented in this paper may be useful to bow makers who are interested in the objective characterization of bows. The need to optimize each bow in spite of variability in wood properties also gives support to a simulation tool based on this model. As the possibility to put the bow under hair tension comes rather late in the making process, simulations could be useful to anticipate the consequences of conception choices on the behavior of the tightened bow, assisting the maker to achieve the right balance between wood properties, taper and camber.

A natural continuation of this work is to establish a link between the mechanical behavior and the playing qualities of a bow. An ongoing subjective study focuses on the influence of camber and hair tension on the perception of the bow by the player.

\section{Acknowledgments}

The study presented in this paper is supported by the French National Research Agency (ANR) within the PAFI project. The authors wish to thank Jean Grunberger, bow maker, for fruitful discussions and for the selection and adjustment of the two bows.

\section{References}

[1] U. Wegst, S. Oberhoff, M. Weller, and M. Ashby. Materials for violin bows. International Journal of Materials Research, 98:1230-1237, 2007. 
[2] J. Regh. Grading methods for pernambuco. Journal of the Violin Society of America, 19(1):3-28, 2004.

[3] G. Lucchi \& Sons, http://www.lucchicremona.com/. last viewed 25 July 2011.

[4] F. Gautier, V. Doutaut, and J.-M. Fouilleul. Lutherie tools : projet collaboratif entre ateliers de lutherie et laboratoires. Musique $\&$ Technique, 4:21-28, 2009.

[5] ANR project PAFI ("Plateforme d'Aide à la Facture Instrumentale"), http://pafi.univlemans.fr/.

[6] A. Askenfelt. Observations on the violin bow and the interaction with the string. STLQPSR, 36(2-3):23-42, 1995.

[7] R. Pitteroff. Contact mechanics of the bowed string. PhD thesis, University of Cambridge, UK, 1995.

[8] P. Carlsson and M. Tinnsten. Geometrical compensation for varying material properties in bows by the use of numerical optimization. Acta Acustica united with Acustica, 93:145-151, 2007.

[9] T. Rossing, editor. The science of string instruments, chapter "Bows, Strings, and Bowing". Springer, New-York, 2010.

[10] F. Ablitzer, J.-P. Dalmont, and N. Dauchez. Static model of a violin bow: influence of camber and hair tension on mechanical behavior. Accepted for publication in the J. Acoust. Soc. Am. (2011).

[11] E. Schoonderwaldt. Mechanics and acoustics of violin bowing: Freedom, constraints and control in performance. PhD thesis, Royal Institute of Technology (KTH), Stockholm, Sweden, 2009.

[12] M. A. Crisfield. Non-linear finite element analysis of solids and structures, volume 2. Wiley, Chichester, 1997.

[13] B. Rolland. The playing parts of the bow: focusing on the stick. Journal of the Violin Society of America, 19(1):201-217, 2002.

[14] A. Grütter. A bow on the couch, http://www.andreasgrutter.nl/. last viewed 25 July 2011. 
[15] I. Brémaud, J. Gril, and B. Thibaut. Anisotropy of wood vibrational properties: dependence on grain angle and review of literature data. Wood Science and Technology, pages $1-20,2010$.

[16] R. Pitteroff and J. Woodhouse. Mechanics of the contact area between a violin bow and a string. part I: Reflection and transmission behaviour. Acta Acustica united with Acustica, 84(3):543-562, 1998.

[17] Joint Committee for Guides in Metrology. Evaluation of measurement data - Supplement 1 to the "Guide to the expression of uncertainty in measurement" - Propagation of distributions using a Monte Carlo method (JCGM 101:2008). Bureau International des Poids et Mesures.

[18] L. Schimleck, C. Espey, C. Mora, R. Evans, A. Taylor, and G. Muniz. Characterization of the wood quality of pernambuco (Caesalpinia echinata lam) by measurements of density, extractives content, microfibril angle, stiffness, color, and nir spectroscopy. Holzforschung, 63:457-463, 2009.

[19] H. Scott. Finding the best bow for the job. Strings, 156:59-63, 2008. 


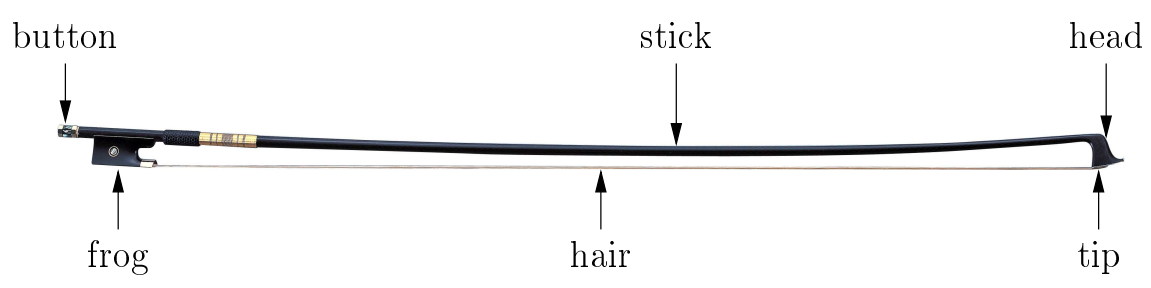

Figure 1: Modern violin bow. 


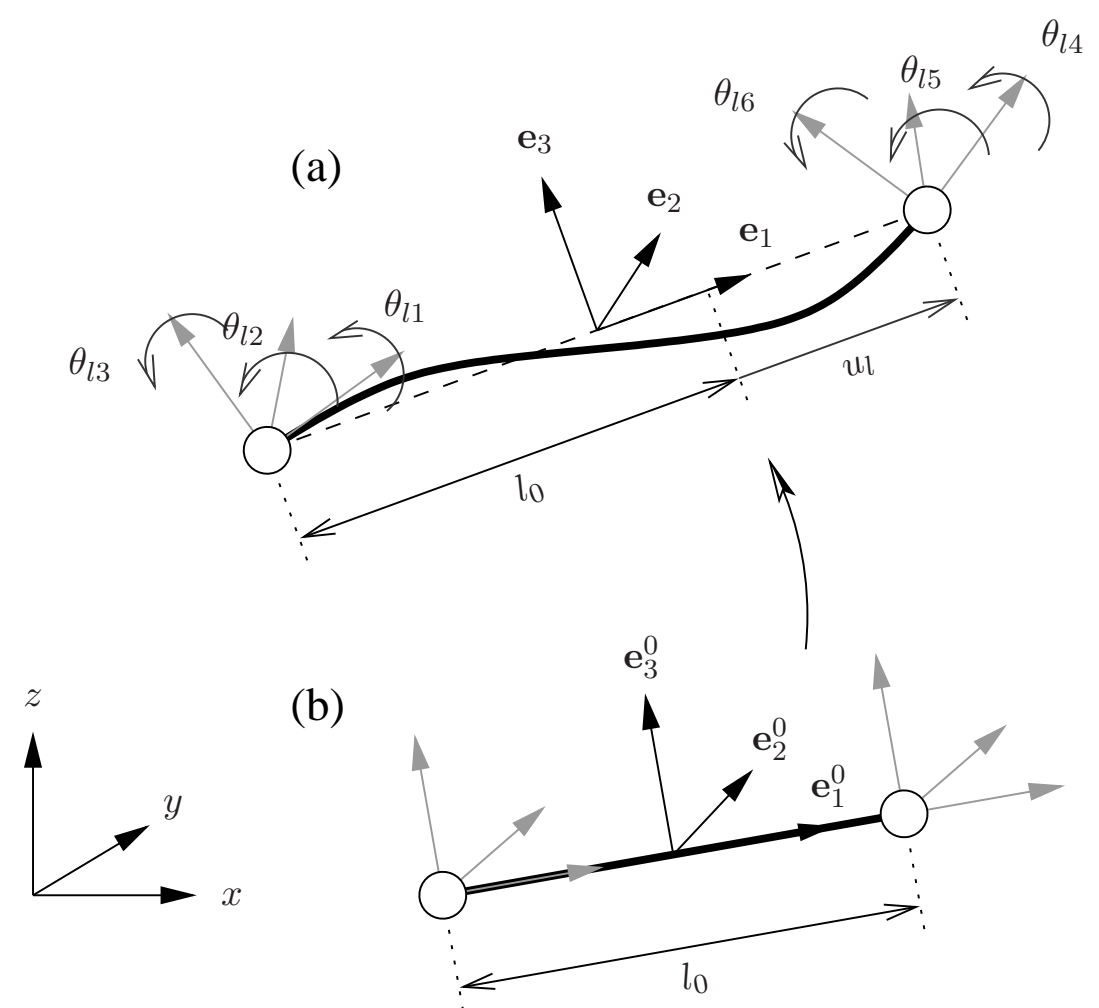

Figure 2: Illustration of the co-rotational approach: (a) current configuration, (b) initial configuration. 


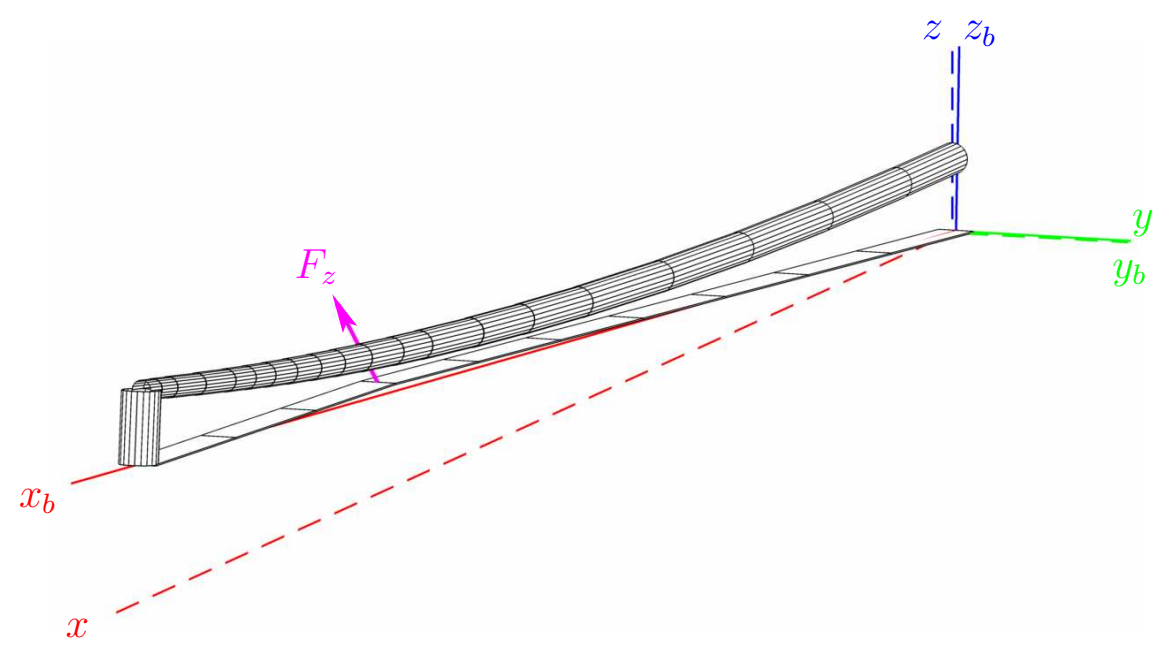

Figure 3: Illustration of the finite element model. The tightened bow is loaded by a force $F_{z}$ inclined relative to the vertical axis of the bow $z_{b}$. The global reference frame $\{x, y, z\}$ is materialized by dashed lines, the reference frame of the bow $\left\{x_{b}, y_{b}, z_{b}\right\}$ by solid lines. 
(0)

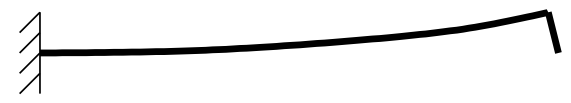

(1)

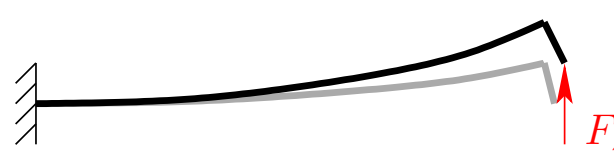

Geometry

$\begin{aligned} & \downarrow \\ F_{z} & \text { of the stick } E_{L}\end{aligned}$

(2)

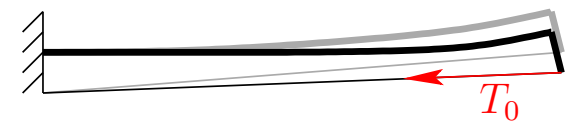

$\checkmark$

Hair tension $T_{0}$

$\checkmark$

(3)

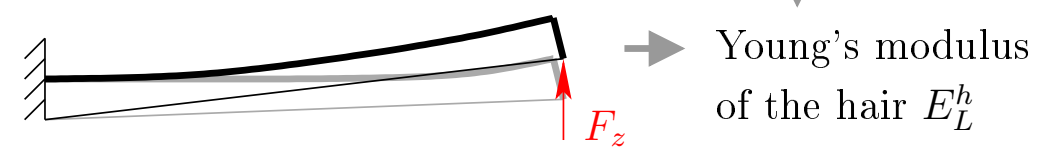

Figure 4: Illustration of the successive steps for the determination of bow parameters. 


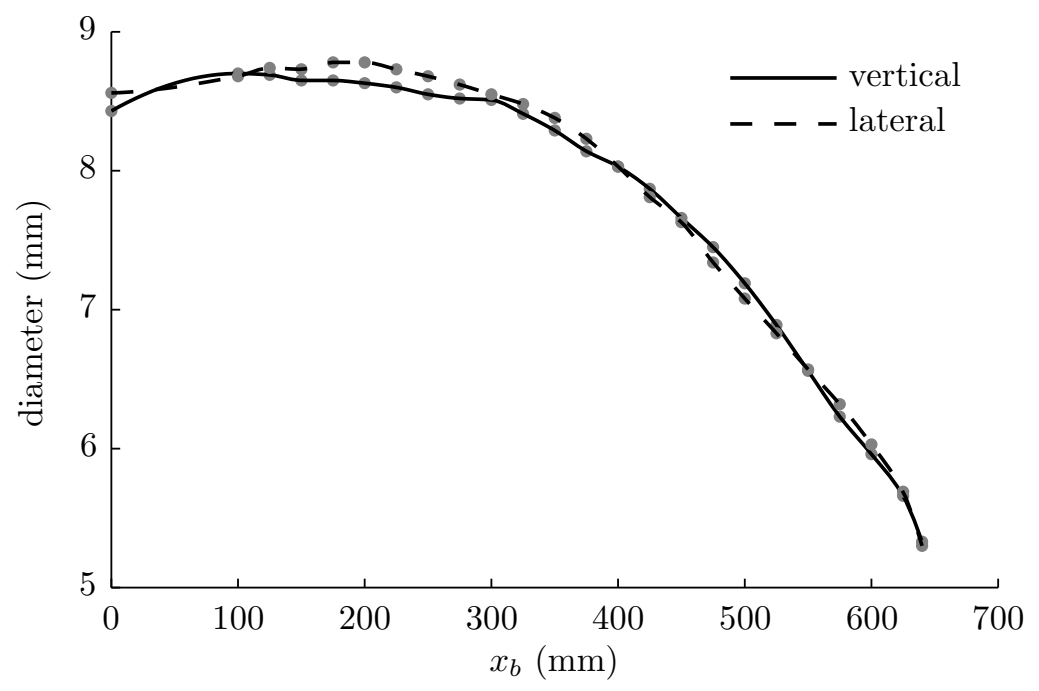

Figure 5: Vertical and horizontal diameters along the stick of bow B1. 


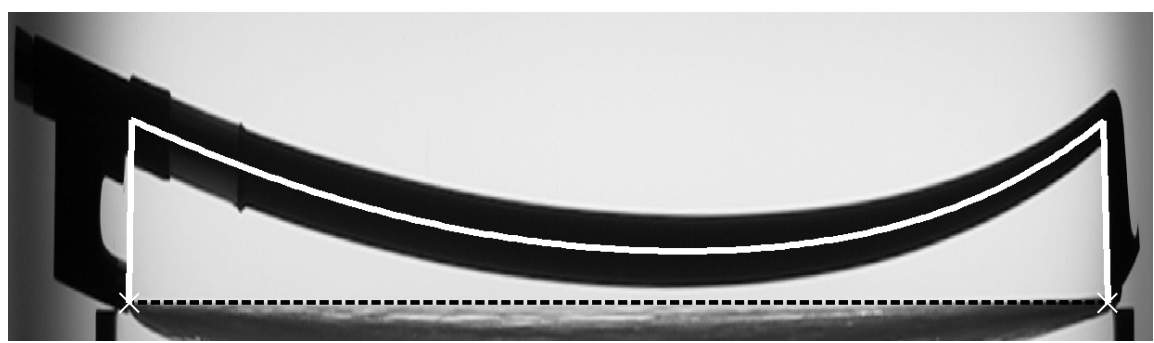

Figure 6: Determination of camber from a picture of the backlighted bow B1 (modified aspect ratio in order to emphasize the curvature along the bow). A 5th order polynom is used here to characterize camber. 

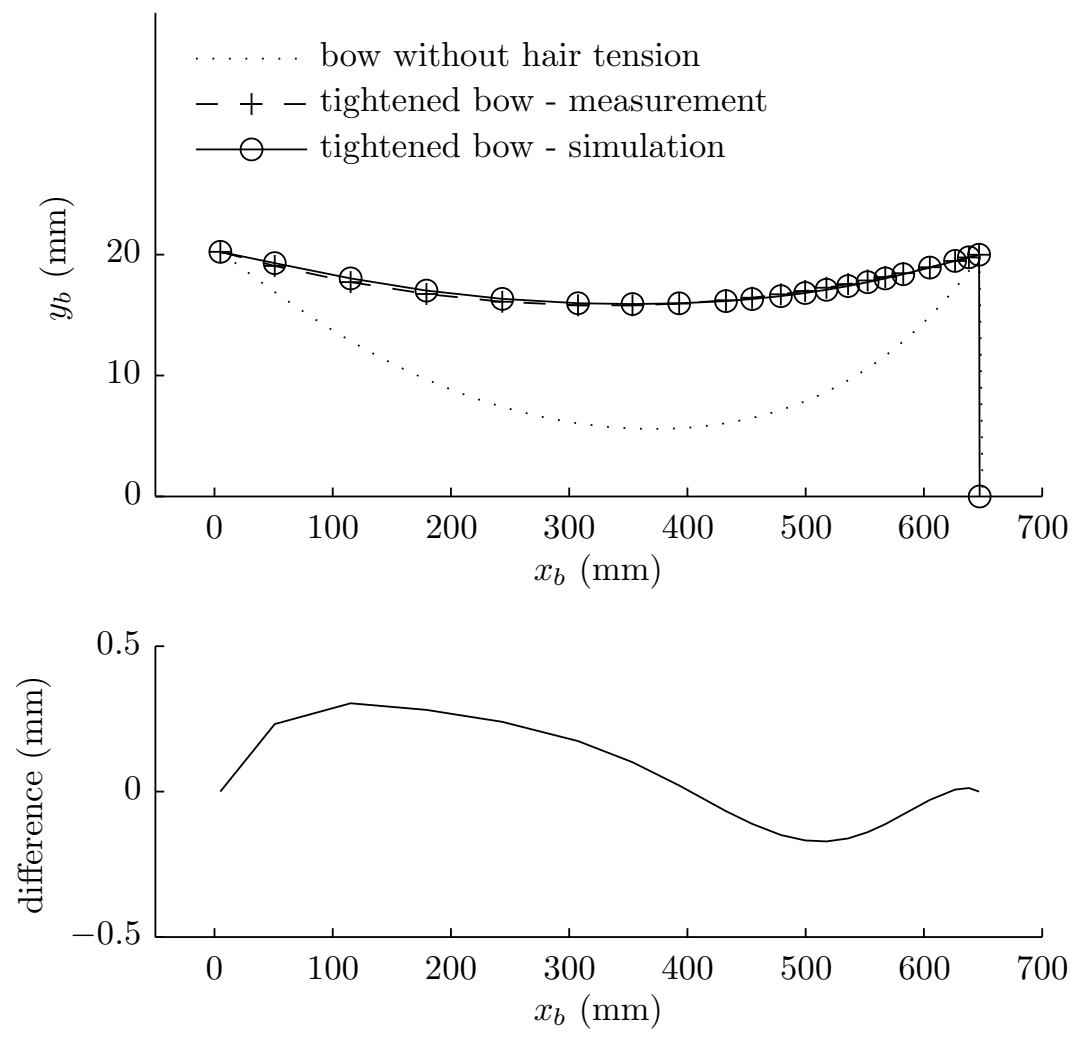

Figure 7: Determination of hair tension $T_{0}$ by an inverse method. The upper plot shows the measured and simulated deflected shape of the stick, compared in the reference frame of the bow, after convergence of the optimization routine. The lower plot shows the residual difference between measurement ad simulation. 


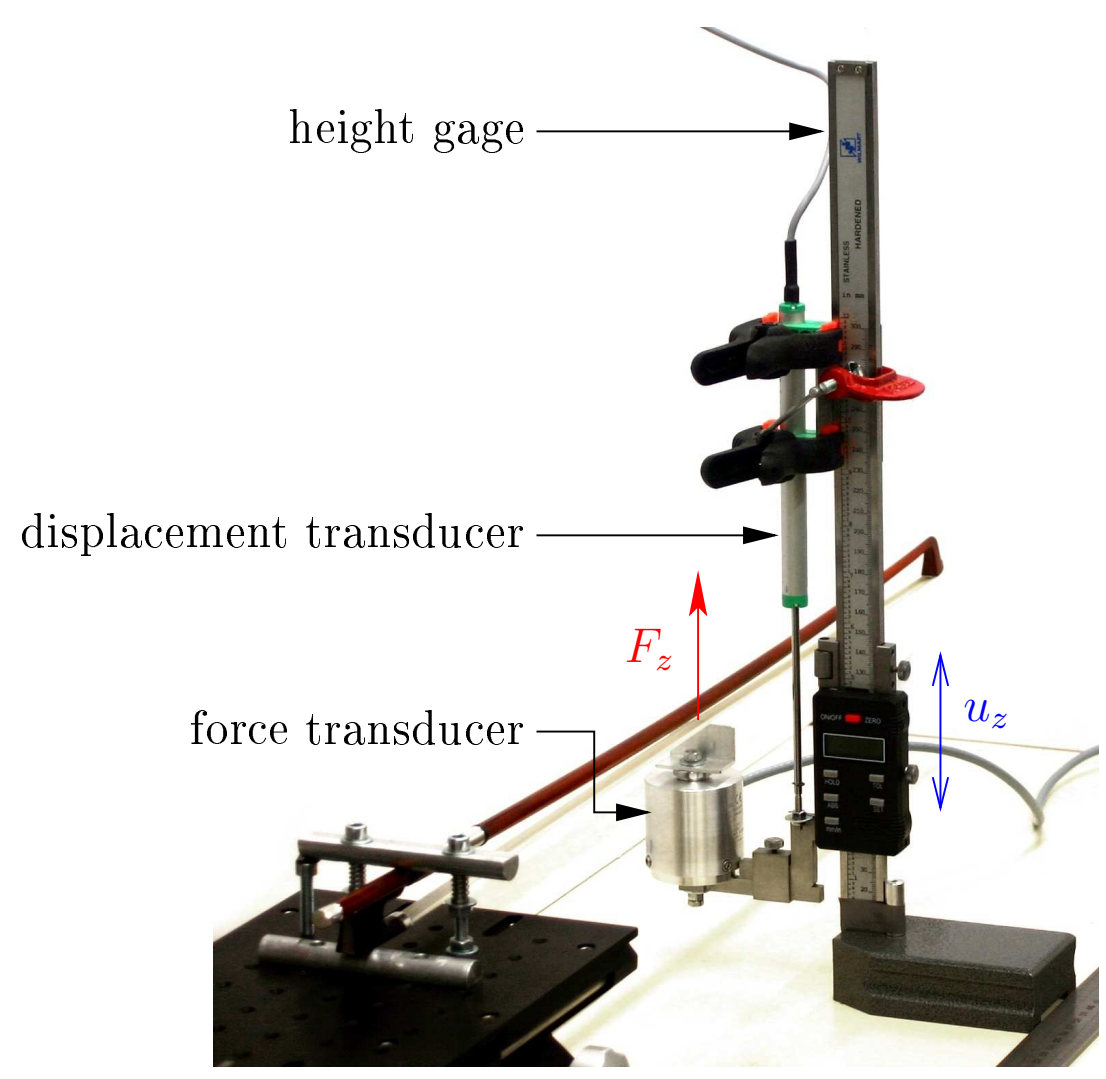

Figure 8: Measurement of vertical compliance on the bow. 


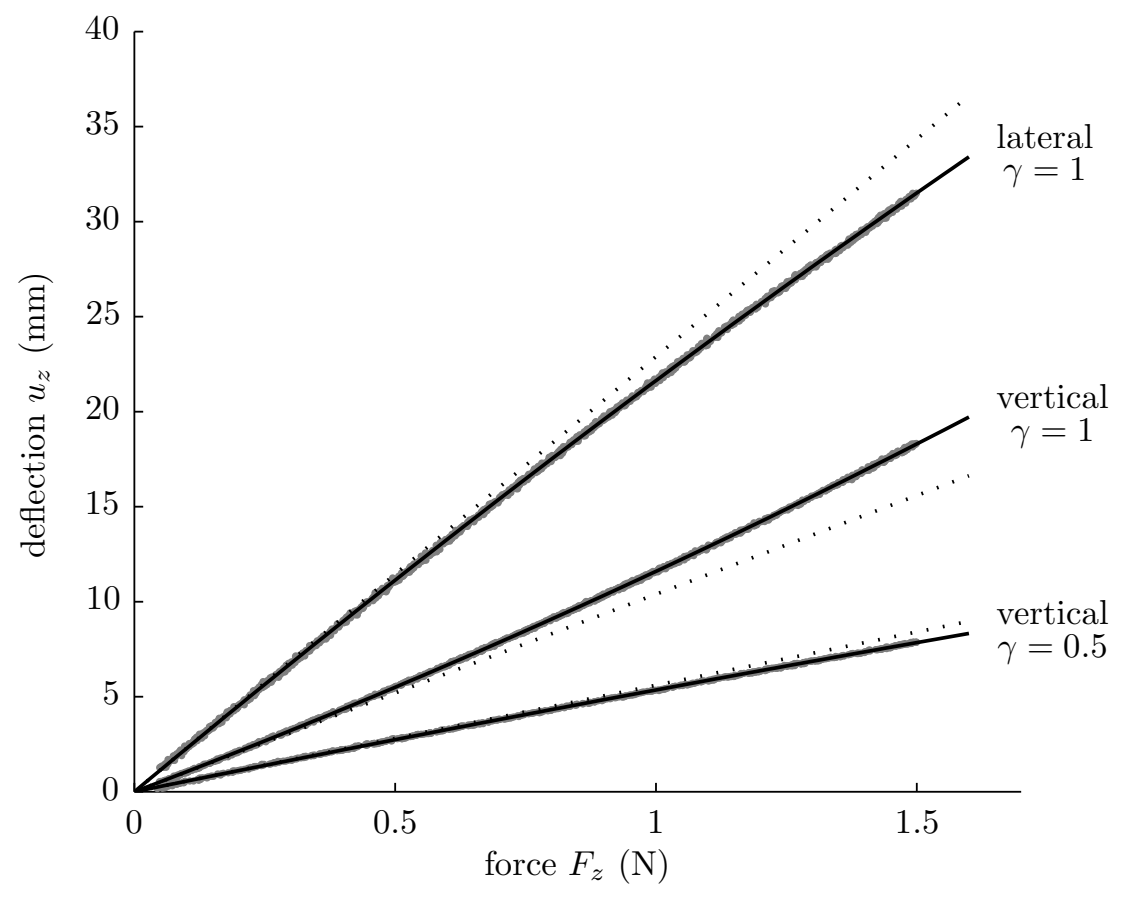

Figure 9: Measured force-deflection curves for three cases of loading on bow B2. Relative abscissa is denoted by $\gamma(\gamma=0$ at the frog, $\gamma=1$ at the tip). Thick lines: experimental data. Thin lines: second order polynom. Dotted lines: linear relationship (tangent at $F_{z}=0 \mathrm{~N}$ ). 


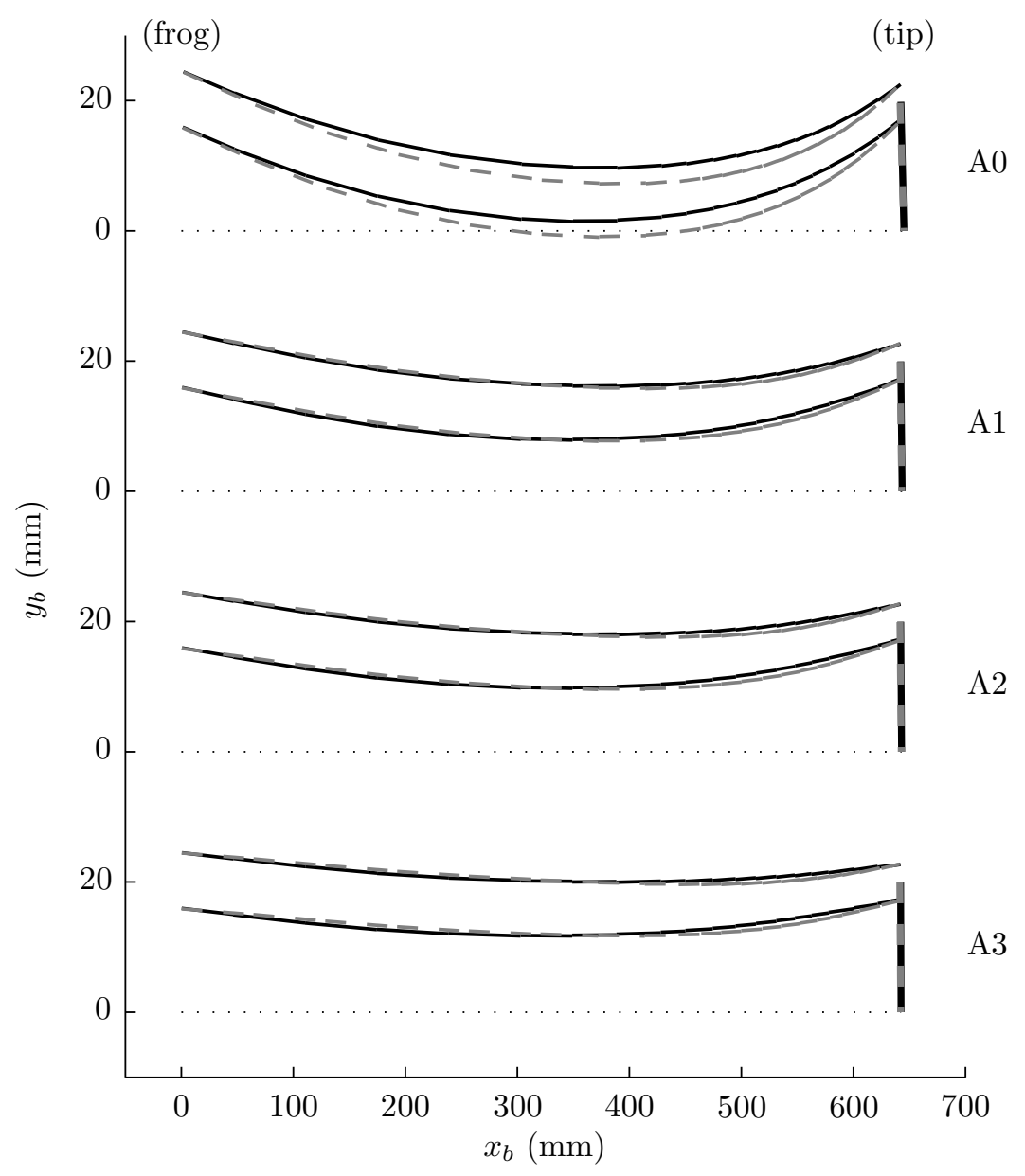

Figure 10: Comparaison between the shapes of bows B1 (-) and B2 (- -) measured without hair tension and for three levels of hair tension (A1, A2, A3), corresponding to three imposed hair-stick distances $(7.8 \mathrm{~mm}, 9.7 \mathrm{~mm}, 11.7 \mathrm{~mm}$, respectively, $\pm 0.1 \mathrm{~mm}$ each). Upper and lower outlines of the stick is represented. 


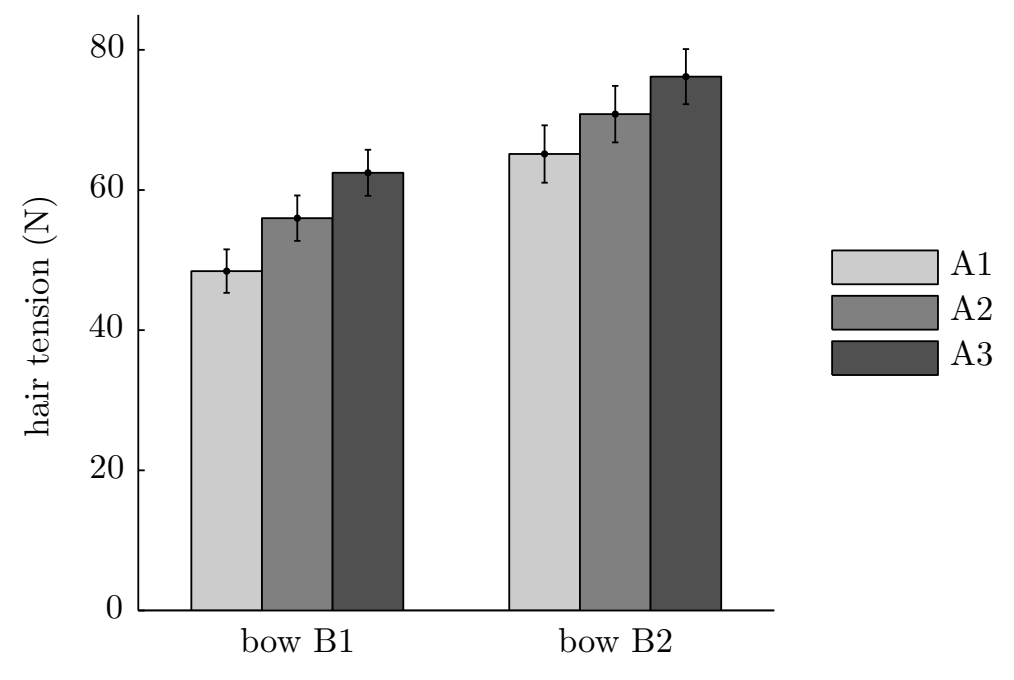

Figure 11: Hair tension corresponding to the three settings of hair-stick distance A1, A2, A3 (see Fig 10). The uncertainties are calculated using the Monte-Carlo method, as described in section 3 . 

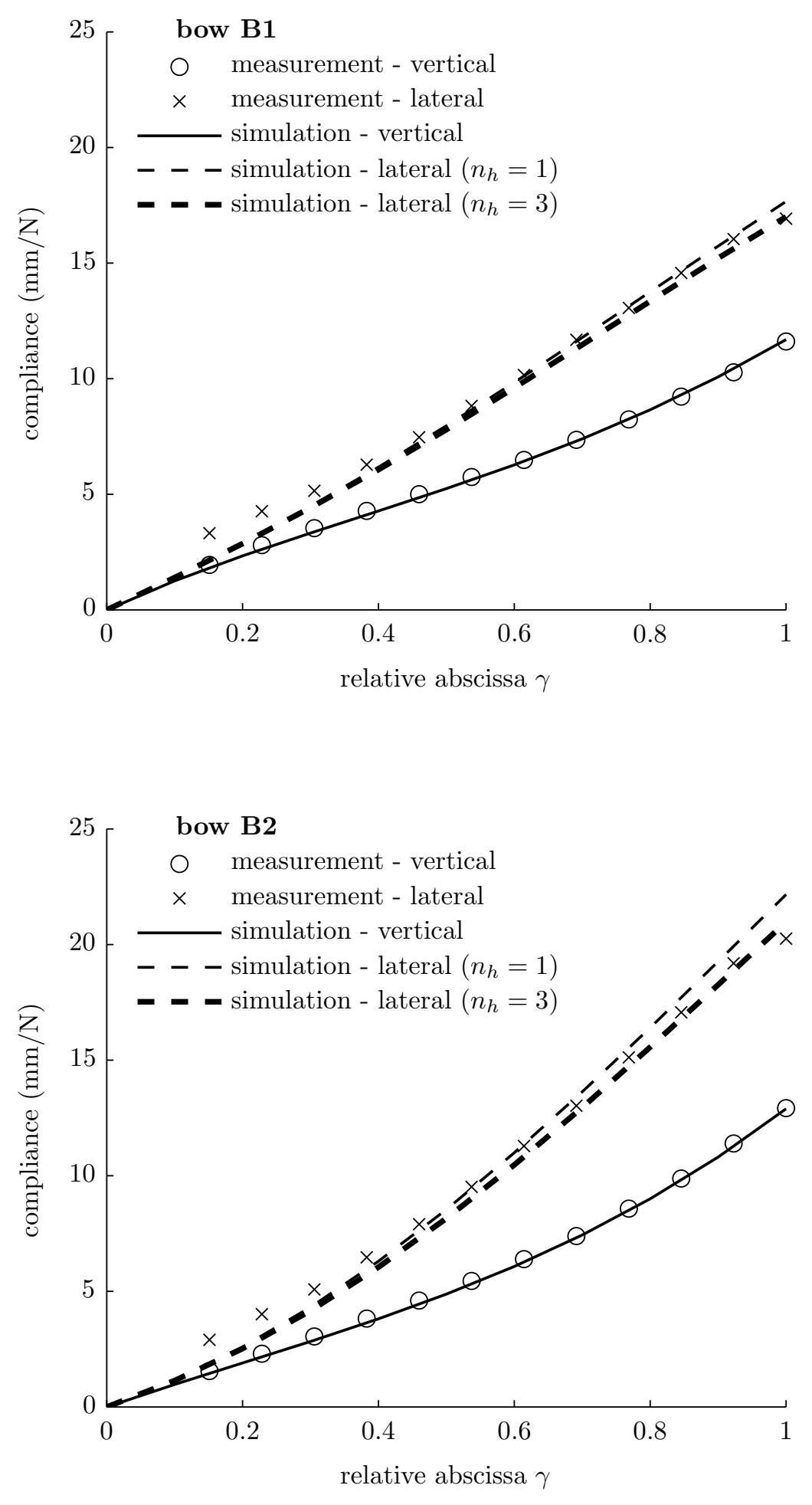

Figure 12: Comparaison between experimental and numerical results on vertical and lateral compliances along the tightened bow, at a bow force of $1 \mathrm{~N}$, for the setting of hair-stick distance A1 (see Fig 10). The number of equivalent hairs used to model the ribbon is denoted by $n_{h}$. 

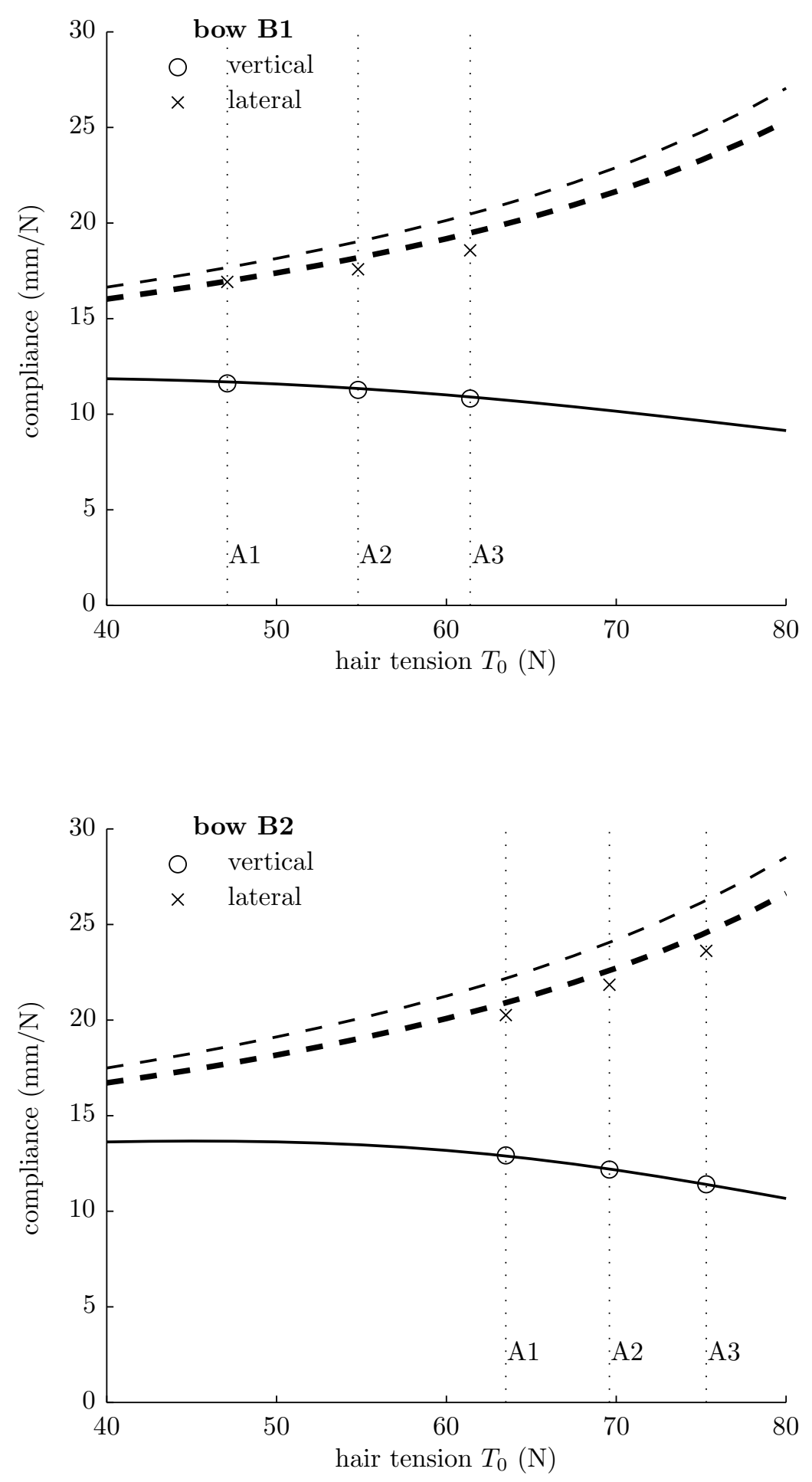

Figure 13: Evolution of vertical and lateral compliances at the tip $(\gamma=1)$ with the hair tension on both bows, at a bow force of $1 \mathrm{~N}$. The experimental values correspond to settings of hair-stick distance A1, A2, A3. Solid lines: simulated vertical compliance. Thin dashed lines: lateral compliance with a single equivalent hair representing the ribbon. Thick dashed lines: lateral compliance with $n_{h}=3$ equivalent hairs. 


\begin{tabular}{lcc}
\hline Input parameter & Value & Uncertainty \\
\hline Vertical diameter $\mathbf{d}_{\mathbf{z}}$ & see Fig. 5 & $\sim 0.1 \mathrm{~mm}$ \\
Horizontal diameter $\mathbf{d}_{\mathbf{y}}$ & see Fig. 5 & $\sim 0.1 \mathrm{~mm}$ \\
Abscissa of the frog $x_{\text {frog }}$ & $0 \mathrm{~mm}$ & $1 \mathrm{~mm}$ \\
Abscissa of the tip $x_{\text {tip }}$ & $645 \mathrm{~mm}$ & $1 \mathrm{~mm}$ \\
Heigth of the frog $y_{\text {frog }}$ & $20 \mathrm{~mm}$ & $1 \mathrm{~mm}$ \\
Heigth of the tip $y_{\text {tip }}$ & $20 \mathrm{~mm}$ & $1 \mathrm{~mm}$ \\
Force at the tip in step $1 F_{z}$ & $1.54 \mathrm{~N}$ & $0.02 \mathrm{~N}$ \\
Force at the tip in step $3 F_{z}$ & $1.54 \mathrm{~N}$ & $0.02 \mathrm{~N}$ \\
\hline
\end{tabular}

Table 1: Input variables needed to determine the bow parameters. 


\begin{tabular}{lcc}
\hline Bow parameter & Value & Uncertainty \\
\hline Young's modulus of the stick $E_{L}$ & $26.8 \mathrm{GPa}$ & $0.5 \mathrm{GPa}$ \\
Hair tension $T_{0}$ & $53.4 \mathrm{~N}$ & $3.0 \mathrm{~N}$ \\
Young's modulus of the hair $E_{L}^{h}$ & $6.5 \mathrm{GPa}$ & $1.4 \mathrm{GPa}$ \\
\hline
\end{tabular}

Table 2: Bow parameters determined by inverse method. 


\begin{tabular}{|c|c|c|}
\hline Characteristic & Bow B1 & Bow B2 \\
\hline Length of the bow ${ }^{(1,2)}$ & $730 \pm 1 \mathrm{~mm}$ & $730 \pm 1 \mathrm{~mm}$ \\
\hline Length of the hair(1) & $647 \pm 1 \mathrm{~mm}$ & $646 \pm 1 \mathrm{~mm}$ \\
\hline Mass & $60.79 \pm 0.02 \mathrm{~g}$ & $60.78 \pm 0.02 \mathrm{~g}$ \\
\hline Center of inertia ${ }^{(1,2)}$ & $253 \pm 1 \mathrm{~mm}$ & $256 \pm 1 \mathrm{~mm}$ \\
\hline Minimum hair-stick distance ${ }^{(1,3)}$ & $1.4 \pm 0.1 \mathrm{~mm}$ & $-1.0 \pm 0.1 \mathrm{~mm}$ \\
\hline Stiffness ${ }^{(3)}$ & 170 & 170 \\
\hline
\end{tabular}

Table 3: Characteristics of the two bows used in the measurements and simulations. ${ }^{(1)}$ without hair tension ${ }^{(2)}$ measured from the origin of the stick ${ }^{(3)}$ a negative value indicates that the stick passes through the hair ${ }^{(4)}$ the value gives the deflection of the stick in microinches simply supported near its ends and loaded in the middle by a $1 \mathrm{lb}$ weight, as measured by the bow maker (generally from 150 , very stiff, to 250 , very flexible) 


\begin{tabular}{cccc}
\hline & $\mathrm{A} 1$ & $\mathrm{~A} 2$ & $\mathrm{~A} 3$ \\
\hline Bow B1 & 1.46 & 1.56 & 1.72 \\
Bow B2 & 1.57 & 1.79 & 2.07 \\
\hline
\end{tabular}

Table 4: Ratio between lateral and vertical compliances at the tip $(\gamma=1)$ for the three settings of hair-stick distance A1, A2, A3, calculated on experimental values (see Fig. 13). 